
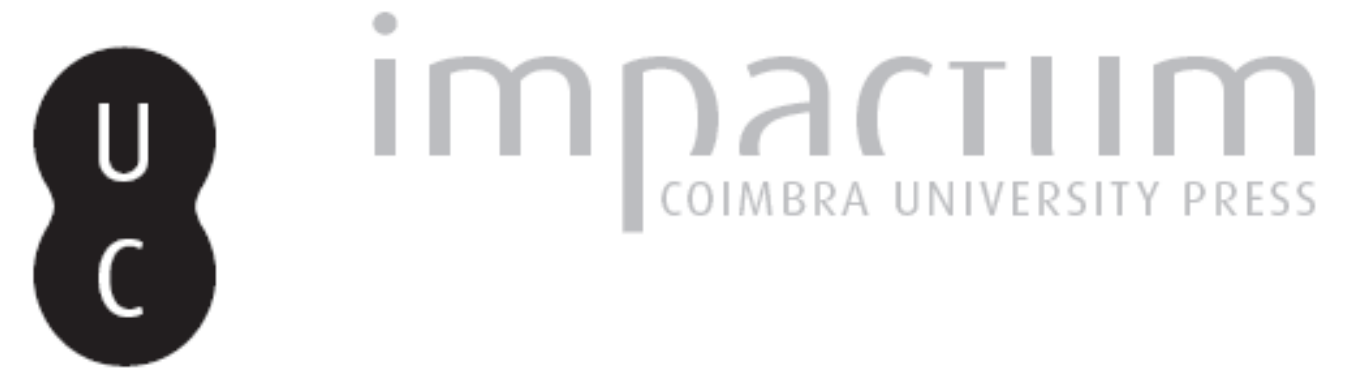

\title{
Production d'amphores et production de salaisons de poisson: rythmes chronologiques sur l'estuaire du Sado
}

Autor(es): $\quad$ Mayet, Françoise; Silva, Carlos Tavares da

Publicado por: Faculdade de Letras da Universidade de Coimbra

URL persistente:

URI:http://hdl.handle.net/10316.2/37863

DOI:

DOI:http://dx.doi.org/10.14195/1647-8657_49_7

Accessed : $\quad$ 26-Apr-2023 10:59:53

A navegação consulta e descarregamento dos títulos inseridos nas Bibliotecas Digitais UC Digitalis, UC Pombalina e UC Impactum, pressupõem a aceitação plena e sem reservas dos Termos e Condições de Uso destas Bibliotecas Digitais, disponíveis em https://digitalis.uc.pt/pt-pt/termos.

Conforme exposto nos referidos Termos e Condições de Uso, o descarregamento de títulos de acesso restrito requer uma licença válida de autorização devendo o utilizador aceder ao(s) documento(s) a partir de um endereço de IP da instituição detentora da supramencionada licença.

Ao utilizador é apenas permitido o descarregamento para uso pessoal, pelo que o emprego do(s) título(s) descarregado(s) para outro fim, designadamente comercial, carece de autorização do respetivo autor ou editor da obra.

Na medida em que todas as obras da UC Digitalis se encontram protegidas pelo Código do Direito de Autor e Direitos Conexos e demais legislação aplicável, toda a cópia, parcial ou total, deste documento, nos casos em que é legalmente admitida, deverá conter ou fazer-se acompanhar por este aviso.

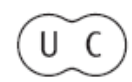


CONIMBRIGA

C)

CN N N

INSTITUTO DE ARQUEOLOGIA

VOLUME XLIX • 2010

FACULDADE DE LETRAS 
FranÇOISE MAYET

Missão Arqueológica Francesa em Portugal

Carlos Tavares da Silva

Centro de Estudos Arqueológicos.MAEDS.Cea.maeds@mail.telepac.pt

\author{
PRODUCTION D'AMPHORES ET PRODUCTION \\ DE SALAISONS DE POISSON: \\ RYTHMES CHRONOLOGIQUES SUR L'ESTUAIRE DU SADO \\ “Conimbriga" XLIX (2010) p. 119-132
}

RESUMO: Os autores, baseando-se nos resultados dos trabalhos que efectuaram no âmbito de projecto sobre a exploração arqueológica do Baixo Sado, identificaram, para esta região, duas grandes fases no processo de produção de preparados piscícolas (e de produção anfórica) durante a época romana, periodização que se pode estender a outras áreas do Sudoeste peninsular, designadamente ao Alentejo Litoral (Sines-Ilha do Pessegueiro). Com efeito, da época claudiana ao final do século II, assiste-se a fase de desenvolvimento económico. Este período interrompe-se bruscamente na passagem para o século seguinte; somente por volta de meados do século III se inicia nova fase de prosperidade, que conhece o seu apogeu no século IV, decai na primeira metade do $\mathrm{V}$ e termina por meados deste último século.

Tem sido difícil definir as causas da oscilação económica negativa ocorrida na transição para o século III. Os autores defendem a hipótese de as destruições registadas e datadas do período considerado terem resultado de um sismo, acompanhado de tsunami, com epicentro no Oceano Atlântico, que teria atingido o litoral do Sudoeste da Península Ibérica.

RÉSUMÉ: En s'appuyant sur les travaux archéologiques qu'ils ont effectués dans la basse vallée du Sado, les auteurs ont identifié pour cette région deux grandes périodes d'activité dans la production des salaisons et des sauces de poisson (et dans la production d'amphores) pendant l'époque romaine. Cette périodisation se retrouve dans d'autres zones

Conimbriga, 49 (2010) 119-132 
du Sud-Ouest péninsulaire, notamment sur le littoral alentéjan (Sines et Pessegueiro). En effet, de l'époque claudienne à la fin du $\mathrm{II}^{\mathrm{e}}$ siècle, on constate partout une phase de grand développement économique, phase brutalement interrompue au début du siècle suivant, caractérisée par de vastes destructions. Ce n'est que vers le milieu du $\mathrm{III}^{\mathrm{e}}$ siècle que s'ouvre une nouvelle période de prospérité qui connut son apogée au $\mathrm{IV}^{\mathrm{e}}$ siècle et se termine aux alentours du milieu du $\mathrm{V}^{\mathrm{e}}$ siècle.

Cette brutale dépression au tournant des $\mathrm{II}^{\mathrm{e}} / \mathrm{III}^{\mathrm{e}}$ siècles n'a jamais été expliquée ; les auteurs défendent l'hypothèse que ces destructions simultanées tout au long du littoral, et du seul littoral, du Sud-Ouest de la Péninsule Ibérique pourraient résulter d'un séisme, accompagné d'un tsunami, ayant son épicentre dans l'océan Atlantique. 


\title{
PRODUCTION D'AMPHORES \\ ET PRODUCTION DE SALAISONS DE POISSON: \\ RYTHMES CHRONOLOGIQUES \\ SUR L'ESTUAIRE DU SADO
}

\begin{abstract}
Du milieu du Ir siècle au milieu du $\mathrm{V}^{\mathrm{e}}$ siècle, les mêmes moments de prospérité et les mêmes moments de régression se sont succédé sur les deux rives de l'estuaire du Sado, aussi bien dans les ateliers d'amphores que dans les établissements de salaisons de poisson. La première période de prospérité, sous le Haut Empire, débute à l'époque claudienne, ou un peu avant, et dure jusqu'à la fin du $\mathrm{II}^{\mathrm{e}}$ siècle. La seconde reprend vers le milieu du $\mathrm{III}^{\mathrm{e}}$ siècle, connaît son apogée au $\mathrm{IV}^{\mathrm{e}}$ siècle, diminue à nouveau dans la première moitié du $\mathrm{V}^{\mathrm{e}}$ siècle pour s'éteindre vers le milieu de ce dernier siècle ; cette seconde période de prospérité correspond à peu de chose près au Bas Empire.

Dans nos différents travaux sur l'exploration archéologique de la basse vallée du Sado, il n'a pas été difficile de trouver les raisons de ces deux moments forts de l'économie de la Lusitanie romaine : présence de marais salants abondants; richesse en poissons des eaux marines et fluviales pour l'industrie des salaisons ; existence de bancs d'argile et de bois de chauffage pour l'industrie céramique. En revanche, nous n'avons pas trouvé d'explication valable pour les deux moments de régression, surtout pour le premier.
\end{abstract}

Conimbriga, 49 (2010) 119-132 


\section{Les établissements de salaisons de poisson}

\section{Tróia}

En étudiant le complexe industriel (usines I/II) ${ }^{1}$ de Tróia, nous avons illustré pour la première fois sur ce site sa destruction au tournant des $\mathrm{II}^{\mathrm{e}}-\mathrm{III}{ }^{\mathrm{e}}$ siècles de notre ère. Deux sondages à l'intérieur de deux bassins ( $\mathrm{n}^{\text {os }} 1$ et 15$)$, condamnés par les dernières restructurations du complexe, ont donné un remplissage et une stratigraphie identiques : sur le sol «nettoyé » de ces deux bassins, reposaient les tuiles de la toiture effondrée de l'usine. Cette couche d'imbrices (7) était surmontée d'un remblai (couches 5 et 6), dans lequel on a découvert des matériaux provenant de la démolition de la maison voisine du propriétaire de l'établissement (stuc peint, sigillée claire A, amphores Dressel 14 et céramique commune). Au-dessus, les couches supérieures (2 à 4) correspondent aux démolitions et constructions postérieures ${ }^{2}$. Parmi le matériel qui nous permet de situer l'abandon de ces deux bassins, nous avons des amphores Dressel 14 lusitaniennes, datables du $\mathrm{II}^{\mathrm{e}}$ siècle, des plats en sigillée claire A (formes Hayes 3, 27 et 14/17) qui ne donnent pas une fourchette chronologique très précise, car ils peuvent dater aussi bien de la fin du $\mathrm{II}^{\mathrm{e}}$ que du début du $\mathrm{III}^{\mathrm{e}}$ siècle. Pour cette raison, nous préférons dater cette destruction du tournant des $\mathrm{II}^{\mathrm{e}}-\mathrm{III}{ }^{\mathrm{e}}$ siècles, l'amphore Dressel 14 de Lusitanie n'apparaissant plus dans les niveaux $\mathrm{du} \mathrm{III}^{\mathrm{e}}$ siècle, à Ostie notamment.

Ce qui nous a frappé dans la fouille de ces deux bassins, c'est de constater que la toiture s'était effondrée en une seule fois et que les tuiles reposaient sur le fond parfaitement nettoyé de ces bassins. Nous n'y avons rencontré aucune arête de poisson, comme si ces bassins, au moment de l'effondrement de la toiture, étaient prêts à fonctionner de nouveau. Cet effondrement survient à la suite d'un événement soudain et inattendu, dans une usine qui n'était pas du tout abandonnée, événement qui a touché tous les secteurs de ce complexe. En effet, le remplissage des deux bassins étudiés signale que la maison du maître, que l'on ne connait pas encore mais qui se trouve certainement sous la dune orientale, a été détruite en même temps que les salles de bassins. D'autre part, l'entrepôt d'amphores situé au nord du complexe a été abandonné à

1 Étienne-Makaroun-Mayet, 1994.

2 Iid., p. 33-36 et fig. 10.

Conimbriga, 49 (2010) 119-132 
ce moment-là : par un sondage et des nettoyages, nous savons que des amphores Dressel 14, prêtes à être remplies de salaisons, sont encore en place le long de ses murs. Cet entrepôt a été surmonté en partie par un mausolée familial, dont on peut dater de ce fait la construction du début du $\mathrm{III}^{\mathrm{e}}$ siècle ; ce mausolée a été postérieurement entouré d'une nécropole qui s'est étendue jusque dans certains bassins abandonnés après le milieu du $\mathrm{V}^{\mathrm{e}}$ siècle.

La conclusion que l'on peut tirer de notre étude de Tróia est qu'un événement inattendu est venu ruiner cet établissement, prêt à fonctionner encore. Il ne s'agit pas d'un phénomène réduit à ce seul établissement ; nous le retrouvons sur d'autres points du même site (voir les derniers travaux d'Inês Vaz Pinto et de Conceição Lopes), et surtout sur d'autres sites du littoral lusitanien et même au-delà.

\section{Autres exemples}

Sans chercher l'exhaustivité ni entrer dans le détail de chacun de ces exemples, nous remarquons une interruption de la production de salaisons de poisson à cette même date (fin $\mathrm{II}^{\mathrm{e}} /$ début $\mathrm{III}^{\mathrm{e}}$ siècle) dans les usines connues de Setúbal, de Sines et de Pessegueiro. Dans Setúbal, la zone « industrielle » qui s'étendait le long de la baie, entre le Largo da Misericórdia et l'actuelle Praça do Bocage, deux établissements de salaisons de poisson ont été fouillés. La fabrique de Travessa de Frei Gaspar $^{3}$ a été construite sur un niveau contenant une maison à péristyle, datée du milieu/troisième quart du $\mathrm{I}^{\text {er }}$ siècle de notre ère ; la fabrique a donc été édifiée dans le dernier quart de ce même siècle. Elle a fonctionné jusqu'aux abords du $\mathrm{III}^{\mathrm{e}}$ siècle, époque à laquelle elle a été abandonnée, au moins partiellement, certains de ces bassins étant utilisés comme dépotoirs. Elle fonctionna à nouveau au $\mathrm{V}^{\mathrm{e}}$ siècle, les bassins recevant un nouveau fond reposant sur les dépotoirs des $\mathrm{III}^{\mathrm{e}}$ et $\mathrm{IV}^{\mathrm{e}}$ siècles. L'établissement fouillé Praça do Bocage ${ }^{4}$ semble avoir été construit dans le dernier quart du $\mathrm{I}^{\mathrm{er}}$ siècle également ; il a fonctionné jusqu'à la fin du $\mathrm{II}^{\mathrm{e}}$ siècle, époque qui a vu son abandon partiel et peut-être même total (avec du matériel comme des sigillées

3 Tavares da Silva-Coelho Soares-Soares, 1986.

4 Tavares da Silva, 1980; Tavares da Silva-Coelho Soares, 1980-1981; Tavares da Silva-Soares, 1986.

Conimbriga, 49 (2010) 119-132 
claires A, des amphores Dressel 14, variante C). Les bassins de cette fabrique servirent de dépotoir aux $\mathrm{III}^{\mathrm{e}}$ et $\mathrm{IV}^{\mathrm{e}}$ siècles (sigillées claires $\mathrm{C}$ et amphores Almagro 51c).

Dans la zone urbaine de Sines, sur le littoral de l'Alentejo, on a fouillé six officines de production de salaisons de poisson ${ }^{5}$. L'évolution de ce petit centre de production présente les deux mêmes phases que les établissements du bas Sado : le plein fonctionnement entre le milieu du I ${ }^{\text {er }}$ siècle et la fin du $\mathrm{II}^{\mathrm{e}}$, se terminant par son abandon ; puis une reprise de la production après le milieu du $\mathrm{III}^{\mathrm{e}}$ siècle sur une zone plus restreinte et avec une subdivision des bassins.

Dans l'île de Pessegueiro ${ }^{6}$, également sur le littoral de l'Alentejo, c'est à partir du début du $\mathrm{III}^{\mathrm{e}}$ siècle que se développe la production de salaisons et sauces de poisson, avec la construction de deux fabriques, sans doute pour faire face au brusque déclin de cette activité dans le centre industriel de Sines.

Il faut naturellement se tourner vers les usines fouillées et publiées récemment pour retrouver l'évolution chronologique de leur activité. Toutefois, quelques fouilles anciennes peuvent être réinterprétées et nous aider à mieux planter la problématique qui nous intéresse. A Baelo Claudia, en Bétique, nous ne connaissons que des usines liées à des maisons à péristyle, mal datées par les fouilles anciennes, ainsi qu'un tout petit établissement, proche du decumanus maximus, dont la chronologie a été établie plus récemment entre l'époque de Claude-Néron et le milieu du III siècle ${ }^{7}$. La fin de l'activité des usines visibles de Baelo a été située à cette dernière date en s'appuyant sur une photographie d'amphores, trouvée par Pierre Paris dans un recoin de l'entrée de la Maison du cadran solaire $^{8}$. Ces amphores semblent pouvoir être rapprochées davantage du type Beltrán IIB que du type Beltrán $72^{9}$. Dans ce cas, maisons et usines liées à ces maisons auraient été détruites vers la fin du $\mathrm{II} / \mathrm{e} e ́ b u t ~ d u ~ I I I^{\mathrm{e}}$ siècle plutôt que vers le milieu du $\mathrm{III}^{\mathrm{e}}$ siècle. Nous retrouvons là, avec des amphores différentes, un " abandon » contemporain de celui des usines lusitaniennes du Sado et de la côte alentéjane. Cette destruction d'une partie de la ville de Baelo Claudia a été d'ailleurs constatée sur

\footnotetext{
Coelho Soares-Tavares da Silva, 2004; Tavares da Silva-Coelho Soares, 2006.

Tavares da Silva-Soares, 1993.

Dardaine-Bonneville, 1980, p. 376-419.

Paris et alii, 1923, fig. 51.

Contrairement à l'interprétation de Sillières, 1995, p. 174.
}

Conimbriga, 49 (2010) 119-132 
d'autres monuments, comme le macellum ${ }^{10}$ dont les boutiques ont été abandonnées dès la fin du $\mathrm{II}^{\mathrm{e}}$ siècle. De même, la basilique voisine ne fonctionna que jusqu'au début du III ${ }^{\mathrm{e}}$ siècle, si ce n'est la fin du $\mathrm{II}^{\mathrm{e}}$ siècle (monnaies de Septime Sévère). Ces constatations ont suggéré que l'on vivait dans des ruines ${ }^{11}$ à partir de ce moment-là ; car ces monuments ont continué à se détruire dans le courant du $\mathrm{III}^{\mathrm{e}}$ siècle. Ils n'ont été ni relevés ni réparés, alors que la vie économique semblait se poursuivre mais sans doute pas avec la même force ; plus tard, au IV ${ }^{\mathrm{e}}$ siècle, des maisons seront construites sur leurs ruines.

Les établissements de salaisons de poisson de la Maurétanie Tingitane sont encore plus mal datés pour l'instant, car les fouilles sont anciennes. Seule l'activité de l'usine de Cotta peut être datée grâce aux céramiques publiées par M. Ponsich et $\mathrm{M}$. Tarradell ${ }^{12}$ : aucune céramique sigillée ne paraît être antérieure à l'époque claudienne (il n'y a aucune sigillée italique par exemple) ni postérieure au $\mathrm{II}^{\mathrm{e}}$ siècle de notre ère (sigillée claire A semblable à celle qui a été trouvée dans les bassins abandonnés de Tróia). Cet établissement aurait été transformé en maison non pas à la fin du $\mathrm{III}^{\mathrm{e}}$ siècle, comme le croyaient ces auteurs, mais au tournant des $\mathrm{II}^{\mathrm{e}} / \mathrm{III} \mathrm{e}^{\mathrm{e}}$ siècles, comme nous l'avons déjà démontré et publié il y a treize ans maintenant ${ }^{13}$.

Nous retrouvons donc dans tout le sud-ouest de la Péninsule Ibérique et le nord du Maroc, depuis le détroit de Gibraltar jusqu'à l'estuaire du Tage peut-être, le même coup d'arrêt à une production très florissante de salaisons de poisson qui a inondé tout l'occident romain pendant les deux premiers siècles de notre ère.

\section{Les fours d'amphores}

Parallèlement à la production de sauces et de salaisons de poisson, nous trouvons la fabrication des amphores qui les exportaient. Si nous regardons l'estuaire du Sado, où nous avons travaillé, nous retrouvons les deux périodes de prospérité déjà constatées sur le site de Tróia. La période du Haut Empire, allant du milieu du Ir à la fin du $\mathrm{II}^{\mathrm{e}}$ siècle,

10 Didierjean-Ney-Paillet, 1986, p. 97-98.

11 Sillières, 1993, p. 147-152.

12 Ponsich-Tarradell, 1965, p. 55-68; Ponsich, 1988, p. 150-159.

13 Étienne-Makaroun-Mayet, 1994, p. 115.

Conimbriga, 49 (2010) 119-132 
correspondant à la production de l'amphore Dressel 14 de Lusitanie ; et la période du Bas Empire, allant du milieu du $\mathrm{III}^{\mathrm{e}}$ au milieu du V $\mathrm{V}^{\mathrm{e}}$ siècle, correspondant à une typologie plus variée d'amphores parmi lesquelles domine 1'amphore Almagro 51c.

Que constatons-nous sur les deux ateliers que nous avons fouillés et publiés?

\section{L'atelier d'Abul}

A Abul A tout d'abord ${ }^{14}$, nous avons découvert une batterie de cinq fours dont la conception, sinon toute la construction, peut être située sous le règne de Tibère, époque des essais devant aboutir à l'amphore Dressel 14 lusitanienne qui est fortement produite et diffusée sous le règne suivant de Claude, soit peu avant le milieu du $\mathrm{I}^{\mathrm{er}}$ siècle. Dans le courant du $\mathrm{II}^{\mathrm{e}}$ siècle, en raison d'une montée progressive du niveau de la mer - montée de 0,80 mètre, constatée par Max Guy dans les sondages qu'il a pratiqués sur le site ${ }^{15}$ - les potiers d'Abul ont lutté contre l'inondation des fosses d'accès et des chambres de chauffe des fours 2 et 3 , les plus profondément creusés et sans communication avec la rive proche pour évacuer les excédents d'eau. Ils ont tout d'abord construit des canalisations destinées à drainer l'eau de l'intérieur des fours vers un puisard extérieur. Lutte vaine, ils ont fini par abandonner ces fours avant la fin de la production de l'amphore Dressel 14. Puis, à la fin du $\mathrm{II}^{\mathrm{e}}$ siècle, quand cessa cette production, seul le four 5 , le plus proche de la berge du fleuve, continuait à fonctionner et ce jusqu'au milieu $\mathrm{du} \mathrm{III}^{\mathrm{e}}$ siècle, juste au moment où commence la grande production de l'amphore Almagro 51c, dans sa variante B. A partir de ce moment-là, les potiers abandonnèrent totalement l'officine d'Abul A pour s'installer huit cents mètres plus à l'ouest, sur le site d'Abul $\mathrm{D}$, où ils ont travaillé jusqu'au milieu du Ve siècle.

Sur le même site d'Abul A, nous avons mis au jour un entrepôt d'où partaient les amphores produites dans cet atelier pour être remplies de salaisons de poisson dans les établissements de Tróia et Setúbal.

14 Mayet-Tavares da Silva, 2002.

15 M. Guy, J. Guy et J. Delézir, dans Mayet et Tavares da Silva, 2000, p. 252.

Conimbriga, 49 (2010) 119-132 
Nous n'avons pas eu le plan complet, sans doute assez grand, de l'édifice primitif, mais nous avons remarqué l'installation d'un dallage de briques au-dessus d'un niveau d'amphores Dressel 14 imbriquées les unes dans les autres et destinées à drainer un terrain de plus en plus souvent inondé par la remontée des eaux. Par les variantes A et B de ces amphores, on peut dater ce dallage du début du $\mathrm{II}^{\mathrm{e}}$ siècle. Cet entrepôt a été détruit à la fin de ce même siècle et grossièrement reconstruit, avec des dimensions beaucoup plus réduites, au moment où il n'y avait plus que le four 5 en fonctionnement.

\section{L'atelier de Pinheiro.}

Sur l'atelier voisin de Pinheiro, nous retrouvons une chronologie comparable. Les deux fours jumelés (fours 1 et 2), excellemment construits, ont cuit essentiellement des amphores Dressel 14 depuis l'époque flavienne. L'un des deux (four 2) a cessé de fonctionner à la fin du II ${ }^{\mathrm{e}}$ siècle en même temps qu'un tout petit four à opercules (four 7), qui était très proche de lui, et en même temps aussi qu'un four à tuiles et à briques (four 8) et que la cuisine commune, tous deux plus proches de la berge ancienne du fleuve. Le four 1 a continué à fonctionner jusque vers le milieu du $\mathrm{III}^{\mathrm{e}}$ siècle puis a été remplacé par deux autres fours (fours 4 et 5), moins bien construits mais sans doute moins profonds et moins facilement inondables, surtout le second plus à l'intérieur et nettement plus haut par rapport au niveau de la mer.

Nous avons cité les ateliers que nous connaissons le mieux, mais l'on pourrait étendre ces conclusions à d'autres sites, comme Porto dos Cacos dans la vallée du Tage, par exemple. Il faudrait voir ce qui s'est passé dans la baie de Cadix, où de très nombreux fours étaient construits près du littoral : là aussi il semble y avoir une certaine rupture entre les amphores du Haut Empire (de l'amphore Dressel 18 jusqu'à l'amphore Beltrán IIB) ${ }^{16}$ et celles du Bas Empire. Malheureusement, la chronologie du fonctionnement des fours est encore trop mal connue. Les fours de Puente Melchor (Puerto Real) n'ont pas encore fait l'objet d'une monographie complète : on y relève deux grands fours qui auraient fonctionné depuis l'époque augustéenne jusqu'à un moment quelque peu imprécis entre les ultimes décennies du $\mathrm{II}^{\mathrm{e}}$ et les premières décennies

16 Pour cette typologie, voir Étienne-Mayet, 2002, p. 110-136.

Conimbriga, 49 (2010) 119-132 
du $\mathrm{III}^{\mathrm{e}}$ siècle $^{17}$. Cette chronologie s'appuie sur un vaste dépotoir qui a fonctionné pendant quatre siècles et sur les fouilles d'Augst. Un troisième four, bien plus petit, aurait cuit des amphores Almagro 51c. Il est évident que la datation finale des deux grands fours n'est pas très précise ; il faudrait savoir s'ils ont été ou non inondés au tournant des $\mathrm{II}^{\mathrm{e}} / \mathrm{III}^{\mathrm{e}}$ siècles, comme cela a été le cas en Lusitanie. La typologie des amphores évolue fortement à partir du $\mathrm{III}^{\mathrm{e}}$ siècle dans les fours de la baie de Cadix, comme en Lusitanie ; la difficulté est de savoir si elle est consécutive au même événement que dans la province voisine.

\section{Conclusions}

Si l'on constate, à Pinheiro comme à Abul, l'abandon progressif de fours en raison de la montée du niveau de la mer, la destruction presque généralisée des fours d'amphores comme des usines de salaisons au tournant des $\mathrm{II}^{\mathrm{e}} / \mathrm{III}^{\mathrm{e}}$ siècles est davantage le reflet d'un événement soudain et catastrophique. Il ne peut s'agir que d'une catastrophe naturelle car nous ne croyons pas qu'une simple crise économique - qui d'ailleurs ne semble pas exister - puisse provoquer une destruction et un abandon aussi généralisés au même moment.

Cette catastrophe naturelle nous paraît devoir être liée à un séisme ou plus exactement à un tsunami consécutif à un tremblement de terre dont l'épicentre aurait été situé dans l'Océan Atlantique. Cette hypothèse nous a paru évidente lorsque nous avons appris qu'un tremblement de terre survenu à l'ouest du cap Saint-Vincent (été 2007) a provoqué un léger tsunami qui a été ressenti jusqu'à la presqu'île de Tróia. S'il y avait eu, il y a dix-huit siècles, un tsunami de l'importance de celui qui a eu lieu dans l'Océan Indien en 2004, tout ce qui était bâti sur le littoral et sur les berges des estuaires pourrait fort bien avoir été détruit comme des maisons, des villages de pêcheurs et même des villes l'ont été en Indonésie, en Thaïlande, à Ceylan et en Inde. A l'époque romaine, ce sont surtout les marais salants, les établissements de salaisons, les bateaux de pêche sans doute, les fours d'amphores, déjà menacés par la remontée naturelle des eaux, qui ont dû disparaître d'un instant à l'autre.

Seul ce type de catastrophe est capable d'expliquer les destructions subites et contemporaines, constatées dans les fouilles. Toutes les usines

17 Garcia Vargas-Lavado Florido, 1995.

Conimbriga, 49 (2010) 119-132 
ne seront pas déblayées et réoccupées postérieurement, comme les usines I/II ; beaucoup sont restées en ruine ou bien ont été réutilisées différemment, celles qui ont survécu se sont trouvées plus petites, avec des capacités de production inférieures. Le traumatisme subi par la population, sans doute en partie décimée, a dû marquer les esprits pour une ou plusieurs générations. C'est à la suite de ces événements qu'a été élevé le mausolée au-dessus de l'entrepôt des usines I/II. A l'intérieur, des tombes et des tombeaux à niches laissent à penser qu'on a dû faire face à de nombreux décès simultanés. Il a fallu reconstituer les marais salants, remettre en activité les usines les moins détruites et les moins proches du littoral, bâtir de nouveaux fours qui ont cuit de nouveaux types d'amphore, sans parler de la reconstitution des flottilles de pêche dont nous ne pouvons retrouver aucune trace. Si les exportations de salaisons de poisson ont repris à partir du milieu du $\mathrm{III}^{\mathrm{e}}$ siècle, il faut attendre le $\mathrm{IV}^{\mathrm{e}}$ siècle pour qu'elles retrouvent un niveau convenable, mais certainement inférieur à celui qu'elles avaient sous le Haut Empire.

Certes, ce n'est qu'une hypothèse que l'on ne pourra sans doute jamais vérifier, mais elle a le mérite d'expliquer comment cette industrie a pu être brutalement décimée alors qu'il n'y a aucune crise économique dans le monde romain. Seuls le littoral du sud-ouest de la Péninsule Ibérique et sans doute aussi celui du nord-ouest du Maroc ont subi le contrecoup de cette catastrophe naturelle. L'économie des régions intérieures a pu ne pas trop en souffrir, même si ces régions n'ont plus reçu de salaisons de poisson pendant un certain temps. Elle a aussi le mérite d'expliquer la lenteur de la reprise de cette activité et les changements qu'elle présente. Cette industrie n'a pas retrouvé son importance des deux premiers siècles de notre ère. Les thons mêmes semblent avoir disparu des salaisons du Bas Empire ; les migrations des bancs de thon ont pu s'éloigner des côtes ibériques à la suite d'une catastrophe de ce genre.

Nous ne pensons pas que l'on puisse proposer une explication semblable pour l'interruption définitive de l'industrie de salaisons au milieu du $\mathrm{V}^{\mathrm{e}}$ siècle. On ne retrouve pas d'effondrement des toitures au fond des bassins, bassins qui contiennent souvent des restes piscicoles. On constate plutôt une interruption des exportations de salaisons et un arrêt des importations de céramiques sigillées, aussi bien sur le littoral que dans les villas de l'intérieur comme São Cucufate ${ }^{18}$. Cette situation,

18 Alarcão-Étienne-Mayet, 1990, p. 47-50.

Conimbriga, 49 (2010) 119-132 
bien différente de celle de la fin du II ${ }^{\mathrm{e}}$ siècle, reflète davantage une crise commerciale et sans doute économique de toute la région.

\section{BIBLIOGRAPHIE}

J. Alarcão, R. Étienne et F. Mayet - Les villas romaines de São Cucufate (Portugal), Paris, 1990.

C. Alonso et L. Ménanteau - Les ports antiques de la côte atlantique de l'Andalousie, du bas Guadalquivir au détroit de Gibraltar. Problématique et étude de cas (Baelo, Tarifa), dans Les structures portuaires de l'Arc atlantique dans l'Antiquité : bilan et perspectives de recherche. Journée d'études, Université de la Rochelle, 24 janvier 2008 (Aquitania. Supplément 18), Bordeaux, 2008.

A. Coelho Soares et C. Tavares da Silva - Novas oficinas de produção de preparados piscícolas na área urbana de Sines. Intervenção arqueológica na Rua Ramos da Costa, dans Musa, 1, Setúbal, 2004, p. 111-122.

S. Dardaine et J.-N. Bonneville - La campagne de fouilles d'octobre 1979 à Belo, dans Mélanges de la Casa de Velázquez, XVI, 1980, p. 376-419.

F. Didierjean, C. Ney et J.-L. Paillet - Belo III. Le macellum, Madrid, 1986.

R. Étienne, Y. Makaroun et F. Mayet, avec la collaboration de G. Hennequin, M. da C. Lopes et C. Tavares da Silva - Un grand complexe industriel à Tróia (Portugal), Paris, 1994.

R. Étienne et F. Mayet - Sauces et salaisons de poisson hispaniques (Trois clés de l'économie de l'Hispanie romaine, II), Paris, 2002.

E. Garcia Vargas et M. L. Lavado Florido - Ânforas alto, médio y bajoimperiales producidas en el alfar de Puente Melchor (= Villanueva, Paso a nível: Puerto Real, Cádiz), dans Spal, Séville, 4, 1995, p. 215-228.

F. Mayet et C. Tavares da Silva, avec la collaboration de J. Costa et Y. Makaroun L'atelier d'amphores de Pinheiro (Portugal), Paris, 1998.

F. Mayet et C. Tavares da Silva, avec la collaboration de Y. Makaroun - Le site phénicien d'Abul (Portugal). Comptoir et sanctuaire, Paris, 2000.

F. Mayet et C. Tavares da Silva, avec la collaboration de J. Costa et Y. Makaroun L'atelier d'amphores d'Abul (Portugal), Paris, 2002.

P. Paris, G. Bonsor, A. Laumonier, R. Ricard et C. de Mergelina - Fouilles de Belo (Bolonia, province de Cadix) (1917-1923). I. La ville et ses dépendances, Bordeaux-Paris, 1923.

M. Ponsich - Aceite de oliva y salazones de pescado. Factores geo-económicos de Bética y Tingitana, Madrid, 1988.

M. Ponsich et M. Tarradell - Garum et industries antiques de salaison dans la Méditerranée occidentale (Bibliothèque de l'École des Hautes Études Hispaniques, XXXVI), Paris, 1965.

Conimbriga, 49 (2010) 119-132 
P. Sillères - Vivait-on dans des ruines au $\mathrm{II}^{\mathrm{e}}$ siècle ap.J.-C. ?, dans Ciudad y comunidad cívica en Hispania (siglos II Y III d.C.) (Collection de la Casa de Velázquez, 40), Madrid, 1993.

P. Sillières - Baelo Cláudia, une cité romaine de Bétique, Madrid, 1995.

C. Tavares da Silva - Escavações arqueológicas na Praça do Bocage: 2000 anos de história, Setúbal, 1980.

C. Tavares da Silva et A. Coelho Soares - A praça do Bocage (Setúbal) na época romana. Escavaões de 1980, dans Setúbal Arqueológica, VI-VII, 1980-1981, p. 249-294.

C. Tavares da Silva et A. Coelho Soares - Escavações arqueológicas no Creiro (Arrábida). Campanha de 1987, dans Setúbal Arqueológica, VIII, 1987, p. 231-237.

C. Tavares da Silva et A. Coelho Soares - Produção de preparados piscícolas na Sines romana, dans Produção e Comércio de Preparados Piscícolas durante a Protohistoria e a Época Romana no Ocidente da Península Ibérica (Homenagem a Françoise Mayet), Setúbal Arqueológica, 13, 2006, p. 101-122.

C. Tavares da Silva, A. Coelho Soares et J. Soares - Intervenção arqueológica na Travessa de Frei Gaspar (Setúbal), dans I Encontro Nacional de Arqueologia Urbana (Setúbal, 1985), Lisbonne, 1986, p. 155-160.

C. Tavares da Silva et J. Soares - Arqueologia de Arrábida, Lisbonne, 1986.

C. Tavares da Silva et J. Soares - Ilha do Pessegueiro, porto romano da costa alentejana, Lisbonne, 1993.

Conimbriga, 49 (2010) 119-132 


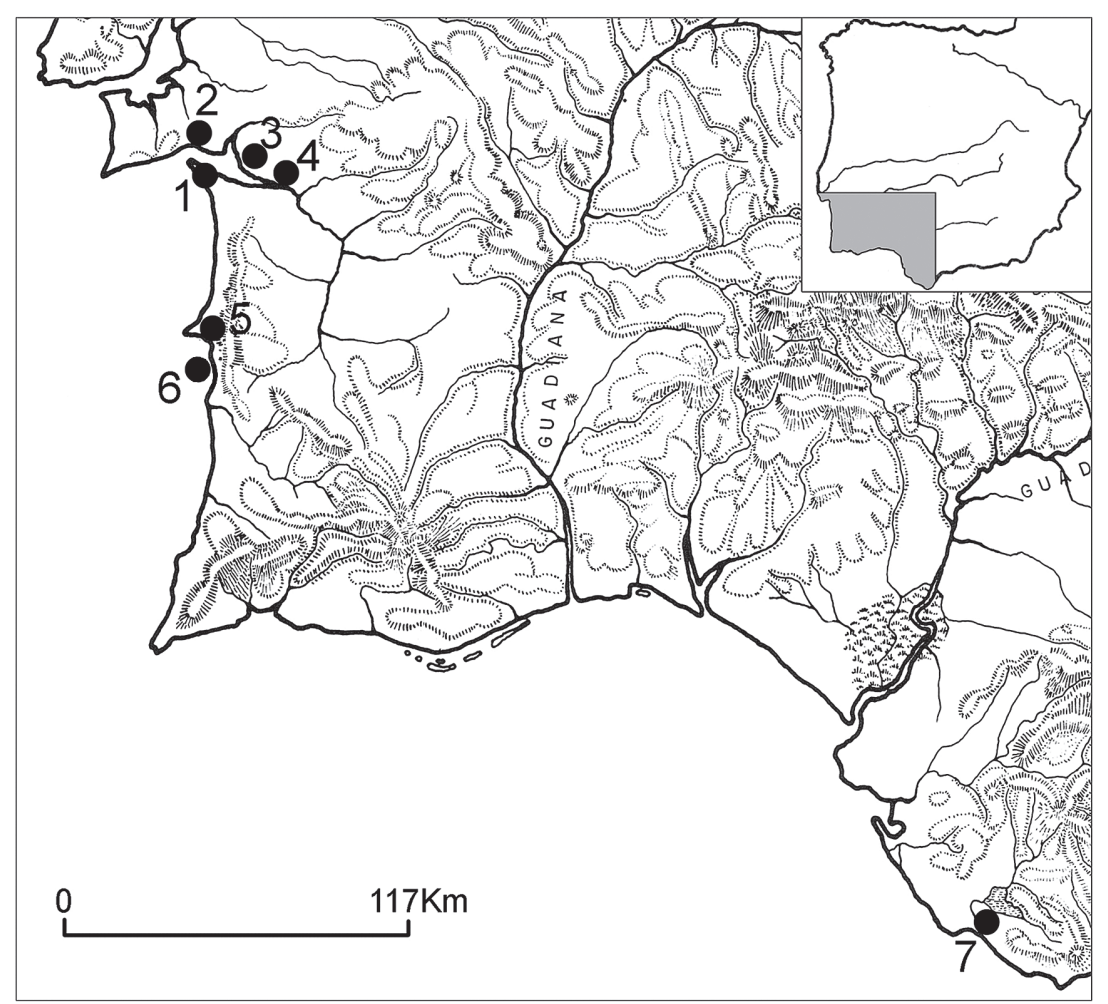

FIG. 1. Localisation des principaux gisements archéologiques cités dans le texte: 1. Tróia; 2. Setúbal; 3. Pinheiro; 4. Abul; 5. Sines; 6. Ile de Pessegueiro, 7. Baelo Claudia. 